\title{
Caracterização morfológica de fungos conidiais decompositores de folhedo provenientes de Cubatão, SP, Brasil
}

\author{
Rosely Ana Piccolo Grandi ${ }^{1,3}$ e Priscila da Silva ${ }^{2}$
}

Recebido: 21.07.2010; aceito: 25.11 .2010

\begin{abstract}
Morphological characterization of litter decomposing conidial fungi from Cubatão, SP, Brazil). This article presents six conidial fungi isolated from leaf litter collected at the municipality of Cubatão, São Paulo State, Brazil. The plant debris were prepared by the successive washing method with sterile distilled water and incubated in moist chambers. The fungi were mounted in permanent slides and deposited in SP Herbarium. The hyphomycetes Dendryphion comosum Wall., Graphium calicioides (Fr.) Cooke \& Massee, Mycoleptodiscus disciformis Matsush., Periconia igniaria E.W. Mason \& M.B. Ellis, Polyschema olivaceum (Ellis \& Everh.) M.B. Ellis and Rhexoacrodictys erecta (Ellis \& Everh.) W.A. Baker \& Morgan-Jones are described and illustrated for the first time to Brazil.
\end{abstract}

Key words: anamorphs, fungal diversity, hyphomycetes

RESUMO - (Caracterização morfológica de fungos conidiais decompositores de folhedo provenientes de Cubatão, SP, Brasil). Este artigo apresenta seis fungos conidiais isolados de folhedo misto coletado no município de Cubatão, estado de São Paulo, Brasil. O folhedo passou pela técnica de lavagem sucessiva e incubação dos detritos em câmaras-úmidas. Os fungos foram montados em lâminas permanentes e encontram-se depositados no Herbário SP. Os hifomicetos Dendryphion comosum Wall., Graphium calicioides (Fr.) Cooke \& Massee, Mycoleptodiscus disciformis Matsush., Periconia igniaria E.W. Mason \& M.B. Ellis, Polyschema olivaceum (Ellis \& Everh.) M.B. Ellis e Rhexoacrodictys erecta (Ellis \& Everh.) W.A. Baker \& Morgan-Jones são descritos e ilustrados pela primeira vez para o Brasil.

Palavras-chave: anamorfos, diversidade de fungos, hifomicetos

\section{Introdução}

Em 1993 pesquisadores do Núcleo de Pesquisa em Micologia do Instituto de Botânica iniciaram um projeto de pesquisa intitulado "Micota dos Vales dos Rios Moji e Pilões, região de Cubatão, SP", com intuito de realizar o levantamento de alguns grupos de fungos nesse município, visto serem escassos trabalhos micológicos na área. Como resultados desse projeto, Pires-Zottarelli (1999) encontrou 56 táxons de fungos zoospóricos (Chytridiomycota e Oomycota), sendo 10 relatados pela primeira vez para o país; Schoenlein-Crusius et al. (2001) relacionaram 40 táxons de fungos anemófilos e Grandi (2004) apresentou 41 táxons de fungos conidiais decompositores da serapilheira identificados até aquela época. Todos os fungos isolados no projeto mencionado, incluindo os dos trabalhos acima citados, foram listados em Schoenlein-Crusius et al. (2006). Salienta-se que Grandi (2004) apresentou a literatura sobre fungos conhecida para a região até aquela data e após o trabalho de Schoenlein-Crusius et al. (2006) nada mais foi publicado sobre fungos para Cubatão.

No trabalho de Schoenlein-Crusius et al. (2006) foram listados 125 fungos anamorfos, muitos já descritos no Brasil. No entanto, restaram seis espécies isoladas da serapilheira sem caracterização morfológica publicada no país. Este trabalho teve por objetivo, portanto, revisar e fornecer a descrição taxonômica dessas seis espécies oriundas de Cubatão.

\section{Material e métodos}

Os espécimes foram isolados a partir de folhedo indeterminado provenientes dos Vales dos Rios Moji

1. Instituto de Botânica, Caixa Postal 3005, 01031-970 São Paulo, SP, Brasil

2. Programa de Pós-graduação em Biodiversidade Vegetal e Meio Ambiente, Instituto de Botânica

3. Autor para correspondência: rapgrandi@uol.com.br 
e Pilões, município de Cubatão, estado de São Paulo, entre março de 1993 a março de 1995. Os dados da região e os procedimentos metodológicos encontramse descritos, em detalhes, em Grandi (2004). Para o isolamento desses fungos utilizou-se a técnica de lavagem sucessiva do folhedo e plaqueamento em câmaras-úmidas, explanada em Grandi \& Gusmão (1998) e Grandi (2004). Materiais fixados em lâminas permanentes, que se encontravam no Herbário SP, foram analisados, revisados e descritos.

As identificações seguiram a taxonomia convencional, isto é, observação da morfologia e obtenção de medidas das estruturas de reprodução e comparação com as espécies descritas. Foram apresentadas descrições e ilustrações para as espécies que não tinham descrição publicada no Brasil. Os nomes dos autores dos fungos estão de acordo com Kirk (2009) e os táxons são apresentados em ordem alfabética.

\section{Resultados e Discussão}

Dendryphion comosum Wallr., Flora Cryptogamica Germaniae 2: 300. 1833.

Figuras 1-2

Conidióforos eretos, vigorosos, septados, lisos, ramificados no ápice, castanho-escuros, 307,4-400,0 $\times 10,3-11,8 \mu \mathrm{m}$ na base. Ramificações curtas e em várias direções, originadas junto aos septos apicais dos conidióforos, lisas, castanhas, com células conidiogênicas no ápice. Células conidiogênicas terminais, integradas, lisas, com cicatrizes evidentes e crescimento simpodial. Conídios tréticos, 2-7-septados apenas transversalmente, cilíndricos, com constrições nos septos, solitários ou em cadeia, verruculosos, castanho-escuros, com extremidades arredondadas e mais claras, frequentemente com cicatrizes, 33,2-59,8 $\times$ 5,9-7,7 $\mu \mathrm{m}$.

Material examinado: BRASIL. São PAULo: Cubatão, Vale do Rio Moji, 23-V-1994, R.A.P. Grandi s.n. (SP251143).

Distribuição geográfica: cosmopolita (Ellis 1971, Hughes 1978, Mercado-Sierra 1984, Abarca et al. 1997, Delgado-Rodríguez \& Mena-Portales 2000).

Dendryphion comosum é a espécie-tipo do gênero (Ellis 1971) e seu teleomorfo é relacionado a Pleosporaceae (Kirk et al. 2008, Kirk 2009). Apenas seis espécies de Dendryphion constam na chave publicada por Siboe et al. (1999), incluindo $D$. comosum, faltando uma revisão mais detalhada do gênero. A espécie possui conídios torulóides, semelhantes aos do gênero Torula; porém os conidióforos, e principalmente as células conidiogênicas deste gênero, são totalmente diferentes (Ellis 1971, Mercado-Sierra 1984). Dendryphion darwinii Siboe, P.M. Kirk \& P.F. Cannon é próxima de $D$. comosum mas apresenta conidióforos pouco ramificados e largura dos conídios maior, entre 6-12 $\mu \mathrm{m}$ (Siboe et al. 1999). Espécie encontrada sobre diversas plantas em decomposição na Europa, nos trópicos e na Nova Zelândia (Ellis 1971, Hughes 1978, Mercado-Sierra \& Heredia 1994, Abarca et al. 1997). Dendryphion comosum foi mencionado, pela primeira vez para o Brasil, em Schoenlein-Crusius et al. (2006) porém sem caracterização morfológica, o que é apresentada aqui.

Graphium calicioides (Fr.) Cooke \& Massee, Grevillea 16(77): 11. $1887 \equiv$ Sporocybe calicioides Fr., Syst. Mycol. 3(2): 343. 1832.

\section{Figura 3}

Sinema escuro, quase preto, indeterminado, com hifas paralelas e monomítico, constituído por conidióforos fortemente adpressos, 439,1 $\mu \mathrm{m}$. compr. Base do sinema alargada, 77,9 $\mu \mathrm{m}$ larg.; ápice de coloração castanho-oliváceo, 13,5 $\mu \mathrm{m}$ larg. Zona fértil apical, globosa, com 110,9 $\mu \mathrm{m}$ diâm., formada por células conidiogênicas e conídios, estes unidos por mucilagem de coloração castanho-clara. Conídios unicelulares, elipsóides ou ovóides, lisos, hialinos, 2,6 × 1,3 $\mu \mathrm{m}$.

Material examinado: BRASIL. SÃo PAULO: Cubatão, Vale do Rio Pilões, 2-VII-1994, R.A.P. Grandi s.n. (SP251147).

Distribuição geográfica: África do Sul (Lee et al. 2004), Austrália (Matsushima 1989, Abarca et al. 1997), Brasil (Schoenlein-Crusius et al. 2006), Costa Rica (Mercado-Sierra et al. 1997), Cuba (MercadoSierra \& Mena-Portales 1995), Hungria (Révay 1985, 1998), México (Abarca et al. 1997), Nova Zelândia (Hughes 1978). Ellis (1971) e Abarca et al. (1997) citam a espécie para a Europa.

Apenas um espécime foi isolado da serapilheira, ficando prejudicada na obtenção das variações das medidas das estruturas. Ellis (1971) aponta, para as medidas dos conídios dessa espécie 1,5-3 × 1-2 $\mu \mathrm{m}$, podendo o sinema atingir até $5 \mathrm{~mm}$ compr. Várias espécies descritas foram consultadas (Fletcher 1975, Matsushima 1975, Hughes 1978) e a que mais se aproximou do material obtido foi $G$. calicioides. Mercado-Sierra (1984) descreveu e ilustrou 
Graphium sp. isolado de detritos vegetais em Cuba, muito semelhante ao presente material. Algumas espécies dentro do gênero passaram por revisão e análise filogenética (Jacobs et al. 2003), mas não se observaram conidióforos e células conidiogênicas anelídicas típicas de Exophiala calicioides (Fr.) G. Okada \& Seifert, nome recentemente aplicado à espécie como informado por Kirk (2009). Optouse, assim, por referenciar a espécie como $G$. calicioides de acordo com Ellis (1971), MercadoSierra et al. (1997), Lee et al. (2004) e outros autores que a isolaram de diversos detritos vegetais. Em Schoenlein-Crusius et al. (2006) consta essa espécie como isolada pela primeira vez no Brasil mas sem descrição taxonômica e por isso é aqui apresentada.

Mycoleptodiscus disciformis Matsush., Matsushima Mycological Memoirs 7: 58. 1993b.

Figura 4-5

Esporodóquio superficial, discóide, com bordas lobadas e irregulares, solitários ou confluentes, com duas camadas de células fortemente aderidas entre si, desprendendo-se do substrato facilmente, castanhoescuro, 81-200 $\mu \mathrm{m}$ diâm. Conidióforos inconspícuos. Células conidiogênicas tipo fiálide, em toda a camada superior, angulares, parede espessa, lisa, castanhas, 5,7-8 $\mu \mathrm{m}$ larg; colaretes distintos e circulares, voltados para a região central do esporodóquio, 1,32,5 $\mu \mathrm{m}$ diâm. Conídios não observados.

Material examinado: BRASIL. São PAULO: Cubatão, Vale do Rio Moji, 10-III-1993, R.A.P. Grandi s.n. (SP251160).

Distribuição geográfica: Brasil (Schoenlein-Crusius et al. 2006) e Peru (Matsushima 1993b).

Apesar dos materiais não apresentarem conídios, as outras características concordaram plenamente com a descrição da espécie (Matsushima 1993b), que foi isolada pela primeira vez sobre folhas em decomposição de "oje" (Ficus insipida Willd.), nome local da planta, em Loreto, Peru. Essa espécie pode ser facilmente distinguida de Mycoleptodiscus brasiliensis Sutton \& Hodges pelo diâmetro do esporodóquio, que tem apenas 30-45 $\mu \mathrm{m}$ diâm. e pela disposição das fiálides (Sutton \& Hodges Junior 1976). Mycoleptodiscus disciformis apresenta distribuição restrita; foi mencionado pela primeira vez para o Brasil por Schoenlein-Crusius et al. (2006) sem descrição, motivo pelo qual faz-se aqui.

Periconia igniaria E.W. Mason \& M.B. Ellis, Mycological Papers 56: 104. 1953.

\section{Figura 6-7}

Conidióforos eretos ou recurvados, ramificados no ápice, lisos, 4-5 septos, base alargada, castanhoescuros, 198,9-289,1 $\times$ 9,2-14,4 $\mu \mathrm{m}$ na base $\mathrm{e}$ 6,7-9,4 $\mu \mathrm{m}$ no ápice. Ramificações apicais com células cilíndricas, lisas, castanhas, onde assentamse as células conidiogênicas globosas ou ovóides, finamente ornamentadas, monoblásticas, castanhas. Conídios globosos, unicelulares, tipicamente com ornamentação em forma de tubérculos, castanhos, 7,7-10,1 $\mu \mathrm{m}$ diâm. Tubérculos 0,5-0,7 $\mu \mathrm{m}$ larg. e 0,7$1,3 \mu \mathrm{m}$ compr.

Material examinado: BRASIL. São PAULO: Cubatão, Vale do Rio Moji, 27-I-1994, R.A.P. Grandi s.n. (SP251165).

Distribuição geográfica: Austrália, Gana, Índia, Jamaica, Estados Unidos da América, Zâmbia (Ellis 1971), Brasil (Schoenlein-Crusius et al. 2006), Hungria (Révay 1998), Ilhas Salomão, Papua-Nova Guiné (Matsushima 1971), Panamá (Morris 1956), Paquistão (Matsushima 1993a), Peru (Matsushima 1993b), Uruguai (Lupo et al. 2001). Ellis (1971) cita-a para a Europa.

Periconia igniaria poderia ser confundida com P. byssoides Pers. mas nesta o diâmetro dos conídios é sempre maior que $10 \mu \mathrm{m}$ (Ellis 1971, Matsushima 1971). Periconia hyderabadensis P. Rag. Rao \& D. Rao tem conídios esféricos com 7-10,5 $\mu \mathrm{m}$ diâm., porém a estrutura conidiogênica tem ramificações unilaterais e células conidiogênicas de várias formas e lisas (Rao \& Rao 1964). O espécime ora descrito possui a fase teleomórfica Didymosphaeria igniaria Booth, um ascomiceto (Booth 1968), mas este não foi encontrado. Pela ocorrência em países de clima tropical e temperado a espécie está amplamente distribuída. Referida em Schoenlein-Crusius et al. (2006) pela primeira vez para o país mas sem descrição, por isso é apresentada aqui.

Polyschema olivaceum (Ellis \& Everh.) M.B. Ellis, More Dematiaceous Hyphomycetes: 372. 1976 三 Clasterosporium olivaceum Ellis \& Everh., Proc. Acad. Nat. Sci. Philad. 1893: 463. 1894. Figura 8

Conidióforos inconspícuos. Células conidiogênicas monotréticas, originadas de pequenas protuberâncias das hifas somáticas, esféricas ou subesféricas e achatadas nos pólos, lisas ou finamente equinuladas, castanho-escuras, 5,1-7,2(-8,1) $\mu \mathrm{m}$ diâm. Conídios fusiforme-alargados, a maioria com 5-7 septos transversais e fortemente constritos nessa 

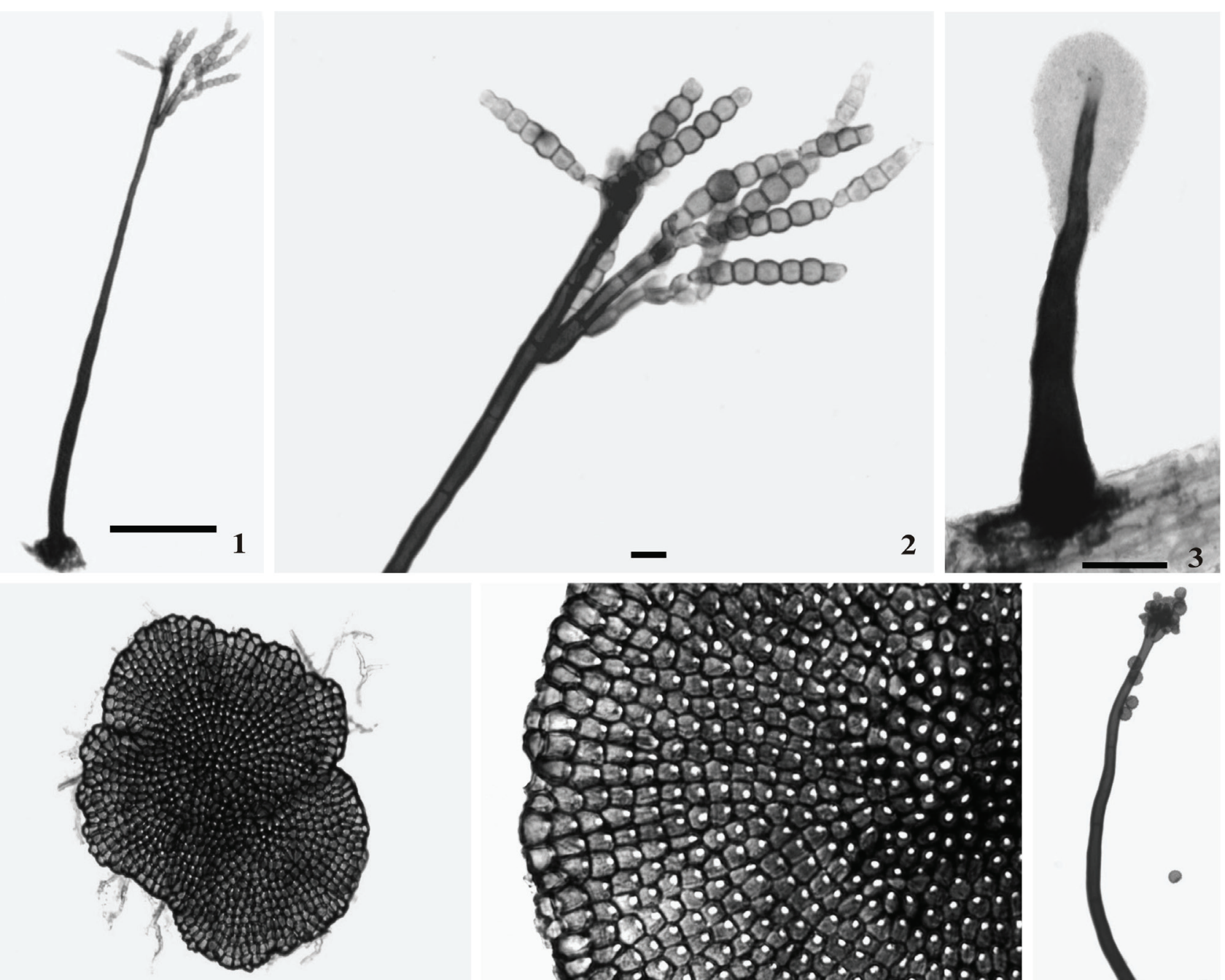

\section{4}
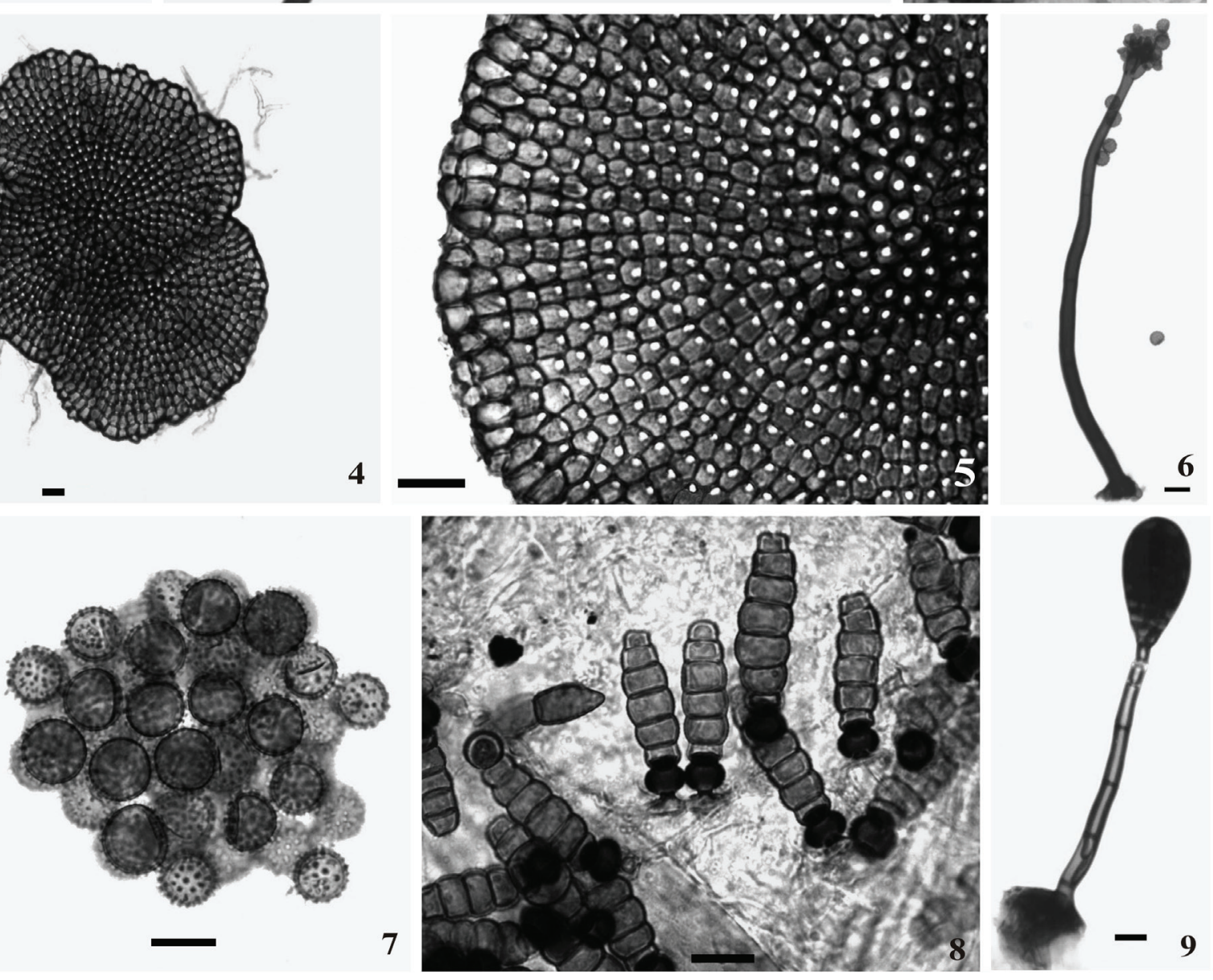

Figuras 1-9. Espécies de fungos conidiais decompositores de folhedo em Cubatão, SP. 1-2. Dendryphion comosum. 1. Hábito. 2. Detalhe do ápice do conidióforo com conídios. 3. Graphium calicioides. 4-5. Mycoleptodiscus disciformis. 4. Esporodóquios confluentes. 5. Parte de um esporodóquio mostrando fiálides, cada uma com um colarete circular. 6-7. Periconia igniaria. 6. Hábito. 7. Conídios com ornamentação típica. 8. Células conidiogênicas e conídios de Polyschema olivaceum. 9. Rhexoacrodictys erecta. Barras de escala: 1,3 = $100 \mu \mathrm{m} ; 2,4-9=10 \mu \mathrm{m}$.

Figures 1-9. Litter decomposing conidial fungi from Cubatão, SP. 1-2. Dendryphion comosum. 1. Habit. 2. Detail of conidiophore apex with conidia. 3. Graphium calicioides. 4-5. Mycoleptodiscus disciformis. 4. Confluent sporodochia. 5. Part of sporodochium showing phialides each with a circular collarette. 6-7. Periconia igniaria. 6. Habit. 7. Conidia with tipical ornamentation. 8. Conidiogenous cells and conidia of Polyschema olivaceum. 9. Rhexoacrodictys erecta. Scale bars: 1, $3=100 \mu \mathrm{m} ; 2,4-9=10 \mu \mathrm{m}$. 
região, finamente equinulados, extremidades apicais arredondadas, castanho-amarelados, 26,3-36,8 $\times$ 6,8-10,7 $\mu \mathrm{m}$.

Material examinado: BRASIL. São PAUlo: Cubatão, Vale do Rio Moji, 26-V-1993, R.A.P. Grandi s.n. (SP251170).

Distribuição geográfica: Brasil (Schoenlein-Crusius et al. 2006) e Estados Unidos da América (Ellis 1976).

Polyschema olivaceum é próximo de Polyschema larviformis (Fairm.) M.B. Ellis mas as células conidiogênicas verrucosas e os conídios bem maiores nesta espécie distinguem os dois táxons (Ellis 1976, Castañeda-Ruiz et al. 2000, Abarca et al. 2006). As ornamentações dos conídios e das células conidiogênicas de $P$. olivaceum são muito sutis, sendo apenas observadas em imersão. A espécie já foi isolada de colmo de milho e do ar, nos Estados Unidos da América (Ellis 1976). Para o Brasil o táxon foi citado, pela primeira vez, na listagem de fungos da região de Cubatão (Schoenlein-Crusius et al. 2006) porém sem descrição, motivo pelo qual é aqui apresentada.

Rhexoacrodictys erecta (Ellis \& Everh.) W.A. Baker \& Morgan-Jones, Mycotaxon 82: 99. $2002 \equiv$ Mystrosporium erectum Ellis \& Everh., J. Mycol. 4: 53.1888 (hoje considerado nomen dubium) $\equiv$ Macrosporium erectum (Ellis \& Everh.) Pound \& Clem., Minn. Bot. Stud. 1 (Bulletin 9): 657.1896 $\equiv$ Acrodictys erecta (Ellis \& Everh.) M.B. Ellis, Mycol. Pap. 79: 12. 1961.

Figura 9

Conidióforos solitários ou em grupos de 2, retos, eretos ou algo flexuosos, sem ramificações, septados, lisos, castanhos, 40,7-81,5 × 5,1-7,1 $\mu \mathrm{m}$ na base. Células conidiogênicas integradas, terminais, monoblásticas, lisas, com proliferação percorrente, castanhas. Conídios solitários, acrógenos, holoblásticos, muriformes, parede espessa, clavadoalargados ou obovóides, lisos, castanhos ou castanhoescuros, opacos, com uma célula basal mais clara contendo resto de parede da célula conidiogênica, 31,1-39,4 × 19,7-25,5 $\mu \mathrm{m}$; célula basal do conídio cilíndrica, lisa, protuberante, 3,8-4,6 $\mu \mathrm{m}$ larg. Liberação rexolítica.

Material examinado: BRASIL. São PAULo: Cubatão, Vale do Rio Pilões, 10-III-1993, R.A.P. Grandi s.n. (SP251126).

Distribuição geográfica: cosmopolita (Baker et al. 2002b).
A espécie era conhecida há muito tempo como Acrodictys erecta (Ellis \& Everh.) M.B. Ellis mas o gênero Acrodictys foi segregado em quatro novos táxons (Baker \& Morgan-Jones 2003, Baker et al. 2001, 2002a, b). A identificação do material em questão segue a proposta de Baker et al. (2002b) sendo a septação dos conídios mais nitidamente percebida na sua parte basal. As mensurações do material concordaram com as apresentadas por Ellis (1971, como Acrodictys erecta), Hyde et al. (1998, como Acrodictys erecta) e Baker et al. (2002b). No entanto, as medidas fornecidas por Matsushima (1975, como Acrodictys erecta) são um pouco menores. Rhexoacrodictys erecta lembra $R$. fuliginosa (B. Sutton) W.A. Baker \& Morgan-Jones mas esta tem conídios menores e pouca septação (Baker et al. 2002b). O táxon ocorre em várias partes de plantas em decomposição, desde os trópicos até as regiões temperadas e foi recentemente mencionado em listagem de hifomicetos ocorrentes na Floresta Nacional de Caxiuanã, no Pará (Gutiérrez et al. 2009). Encontrava-se no Herbário SP como A. erecta e foi mencionado, pela primeira vez para o Brasil, em Schoenlein-Crusius et al. (2006) sem descrição taxonômica, motivo pelo qual é fornecida aqui.

\section{Agradecimentos}

As autoras agradecem à Pesquisadora Marina Capelari pela permissão de uso de sua biblioteca particular.

\section{Literatura citada}

Abarca, G.H., Mena-Portales, J., MercadoSierra, A. \& Estebanez, M.R. 1997. Tropical Hyphomycetes of Mexico. II. Some species from the Tropical Biology Station "Los Tuxtlas", Veracruz, Mexico. Mycotaxon 64: 203-223.

Abarca, G.H., Castañeda-Ruíz, R., BecerraHernández, C.I. \& Mota, R.M.A. 2006. Contribución al conocimiento de los hongos anamorfos saprobios del estado de Tabasco. I. Revista Mexicana de Micología 23: 53-62.

Baker, W.A. \& Morgan-Jones, G. 2003. Notes on Hyphomycetes. XCI. Pseudoacrodictys, a novel genus for seven taxa formerly placed in Acrodictys. Mycotaxon 85: 371-391.

Baker, W.A., Partridge, C. \& Morgan-Jones, G. 2001. Notes on Hyphomycetes. LXXXI. Acrodictyella obovata, a new lignicolous, 
dematiaceous genus and species collected in Alabama. Mycotaxon 78: 29-35.

Baker, W.A., Partridge, C. \& Morgan-Jones, G. 2002a. Notes on Hyphomycetes. LXXXV. Junewangia, a genus in which to classify four Acrodictys species and a new taxon. Mycotaxon 81: 293-319.

Baker, W.A., Partridge, C. \& Morgan-Jones, G. 2002b. Notes on Hyphomycetes. LXXXVII. Rhexoacrodictys, a new segregate genus to accommodate four species previously classified in Acrodictys. Mycotaxon 82: 95-113.

Booth, C. 1968. Didymosphaeria igniaria sp. nov., the perfect state of Periconia igniaria. Transactions of the British Mycological Society 51: 803-805.

Castañeda-Ruíz, R.F., Decock, C., Saikawa, M., Gené, J. \& Guarro, J. 2000. Polyschema obclaviformis sp. nov., and some new records of hyphomycetes from Cuba. Cryptogamie, Mycologie 21: 215-220.

Delgado-Rodríguez, G. \& Mena-Portales, J. 2000. Diversidad y distribución geográfica de los hifomicetos de la Reserva de la Biosfera Sierra del Rosario (Cuba). Boletín de la Sociedad Micológica de Madrid 25: 59-71.

Ellis, M.B. 1971. Dematiaceous Hyphomycetes. Commonwealth Mycological Institute, London,

Ellis, M.B. 1976. More Dematiaceous Hyphomycetes. Commonwealth Mycological Institute, London.

Fletcher, H.J. 1975. Conidiophore morphology in Graphium putredinis. Transactions of the British Mycological Society 64: 67-71.

Grandi, R.A.P. 2004. Anamorfos da serapilheira nos Vales dos Rios Moji e Pilões, município de Cubatão, São Paulo, Brasil. Hoehnea 31: 225238.

Grandi, R.A.P. \& Gusmão, L.F.P. 1998. A técnica da lavagem sucessiva de substratos de plantas como subsídio para estudos da associação fungo/substrato e diversidade de Hyphomycetes nos ecossistemas. In: S. Watanabe (ed.). Anais do IV Simpósio de Ecossistemas Brasileiros, São Paulo, ACIESP, São Paulo, v. 3, pp. 80-90.

Gutiérrez, A.H., Monteiro, J.S. \& Sotão, H.M.P. 2009. Hifomicetos associados a palmeiras na Floresta Nacional de Caxiuanã. In: P.L.B. Lisboa (org.). Caxiuanã: desafios para a conservação de uma Floresta Nacional na Amazônia. Museu Paraense Emílio Goeldi, Belém, pp. 397-405.
Hughes, S.J. 1978. New Zealand Fungi 25. Miscellaneous species. New Zealand Journal of Botany 16: 311-370.

Hyde, K.D., Goh, T.K. \& Steinke, T.D. 1998. Fungi on submerged wood in the Palmiet River, South Africa. South African Journal of Botany 64: 151-162.

Jacobs, K., Kirisits, T. \& Wingfield, M.J. 2003. Taxonomic re-evaluation of three related species of Graphium, based on morphology, ecology and phylogeny. Mycologia 95: 714-727.

Kirk, P.M. 2009. Index Fungorum. (acesso em 16.08.2009).

Kirk, P.M., Cannon, P.F., Minter, D.W. \& Stalpers, J.A. 2008. Dictionary of the Fungi. 10 ed. CAB International, Wallingford.

Lee, S., Mel'nik, V., Taylor, J.E. \& Crous, P.W. 2004. Diversity of saprobic hyphomycetes on Proteaceae and Restionaceae from South Africa. Fungal Diversity 17: 91-114.

Lupo, S., Tiscornia, S. \& Bettucci, L. 2001. Endophytic fungi from flowers, capsules and seeds of Eucalyptus globulus. Revista Iberoamericana de Micología 18: 38-41.

Matsushima, T. 1971. Microfungi of the Solomon Islands and Papua-New Guinea. Publicado pelo autor. Kobe.

Matsushima, T. 1975. Icones Microfungorum a Matsushima Lectorum. Publicado pelo autor, Kobe.

Matsushima, T. 1989. Matsushima Mycological Memoirs n. 6. Matsushima Fungus Collection. Publicado pelo autor, Kobe.

Matsushima, T. 1993a. List of microfungi from Pakistan soils. Cryptogamic Flora of Pakistan 2: 43-63.

Matsushima, T. 1993b. Matsushima Mycological Memoirs n. 7. Matsushima Fungus Collection. Publicado pelo autor, Kobe.

Mercado-Sierra, A. 1984. Hifomicetes Demaciáceos de Sierra del Rosario, Cuba. Academia de Ciencias de Cuba, La Habana.

Mercado-Sierra, A. \& Heredia, G. 1994. Hyphomycetes asociados a restos vegetales en el estado de Veracruz, México. Revista Mexicana de Micología 10: 33-48.

Mercado-Sierra, A. \& Mena-Portales, J. 1995. Hifomicetes dematiáceos de tres provincias orientales de Cuba. Revista Iberoamericana de Micología 12: 101-107. 
Mercado-Sierra, A., Gené, J. \& Guarro, J. 1997. Some Costa Rican Hyphomycetes. II. Mycotaxon 64: 7-15.

Morris, E.F. 1956. Tropical fungi imperfecti. Mycologia 48: 728-737.

Pires-Zottarelli, C.L.A. 1999. Fungos zoospóricos dos Vales dos Rios Moji e Pilões, região de Cubatão, SP. Tese de Doutorado, Universidade Estadual Paulista, Rio Claro.

Rao, P.R. \& Rao, D. 1964. The genus Periconia from India. Mycopathology et Mycology Applicata 22: 285-310.

Révay, Á. 1985. Dematiaceous Hyphomycetes inhabiting forest debris in Hungary. I. Studia Botanica Hungarica 18: 65-71.

Révay, Á. 1998. Review of the Hyphomycetes of Hungary. Studia Botanica Hungarica 27/28: 5-74.
Schoenlein-Crusius, I.H., Trufem, S.F.B., Grandi, R.A.P., Milanez, A.I., Pires-Zottarelli, C.L.A. 2001. Airborne fungi in the region of Cubatão, São Paulo State, Brazil. Brazilian Journal of Microbiology 32: 61-65.

Schoenlein-Crusius, I.H., Milanez, A.I., Trufem, S.F.B., Pires-Zottarelli, C.L.A., Grandi, R.A.P., Santos, M.L. \& Giustra, K.C. 2006. Microscopic fungi in the Atlantic Rainforest in Cubatão, São Paulo, Brazil. Brazilian Journal of Microbiology 37: 267-275.

Siboe, G.M., Kirk, P.M. \& Cannon, P.F. 1999. New dematiaceous Hyphomycetes from Kenyan rare plants. Mycotaxon 73: 283-302.

Sutton, B.C. \& Hodges Junior, C.S. 1976. Eucalyptus microfungi: Mycoleptodiscus species and Pseudotracylla gen. nov. Nova Hedwigia 27: 693-700. 